\title{
Experimental investigation of strong field trident production
}

\author{
J. Esberg, ${ }^{1}$ K. Kirsebom, ${ }^{1}$ H. Knudsen, ${ }^{1}$ H. D. Thomsen, ${ }^{1}$ E. Uggerh $\varnothing j,{ }^{1}$ U. I. Uggerh $\varnothing j,{ }^{1}$ P. Sona, ${ }^{2}$ A. Mangiarotti, ${ }^{3}$ \\ T. J. Ketel, ${ }^{4}$ A. Dizdar, ${ }^{5}$ M. M. Dalton, ${ }^{6}$ S. Ballestrero, ${ }^{7}$ and S. H. Connell ${ }^{7}$
}

(CERN NA63)

\author{
${ }^{1}$ Department of Physics and Astronomy, University of Aarhus, Denmark \\ ${ }^{2}$ University of Florence, Florence, Italy \\ ${ }^{3}$ Universidade de Coimbra, Portugal \\ ${ }^{4}$ Free University, Amsterdam, The Netherlands \\ ${ }^{5}$ University of Istanbul, Istanbul, Turkey \\ ${ }^{6}$ University of Virginia, Charlottesville, Virginia, USA \\ ${ }^{7}$ University of Johannesburg, Johannesburg, South Africa
}

(Received 2 July 2009; revised manuscript received 18 August 2010; published 6 October 2010)

\begin{abstract}
We show by experiment that an electron impinging on an electric field that is of critical magnitude in its rest frame, may produce an electron-positron pair. Our measurements address higher-order QED, using the strong electric fields obtainable along particular crystallographic directions in single crystals. For the amorphous material our data are in good agreement with theory, whereas a discrepancy with theory on the magnitude of the trident enhancement is found in the precisely aligned case where the strong electric field acts.
\end{abstract}

DOI: 10.1103/PhysRevD.82.072002

PACS numbers: 12.20.Fv, 41.60.- m, 41.75.Ht

\section{INTRODUCTION}

Events where an electron produces a pair in the interaction with a nucleus of charge $Z e$ may proceed either through the sequential process where the electron emits a photon that in turn converts into a pair, or directly where the intermediate stage contains a virtual photon. Both types of events $e^{-}+Z \rightarrow e^{-}+Z+e^{-}+e^{+}$ (where $Z$ symbolizes the presence of the nucleus) were originally termed "tridents" because of the observed threeprong track [1,2], but are also referred to as "electroproduction". The same type of event will appear when the nucleus is replaced by an electric field $\mathcal{E}$ of sufficient strength, $e^{-}+\mathcal{E} \rightarrow e^{-}+\mathcal{E}+e^{-}+e^{+}$.

The scale of the electric field relevant in the context of trident events is of the order of the critical field $\mathcal{E}_{0}=$ $m^{2} c^{3} / e \hbar=1.32 \times 10^{16} \mathrm{~V} / \mathrm{cm}$, corresponding to a magnetic field $B_{0}=4.41 \times 10^{9} \mathrm{~T}$. In such fields, an energy equal to the rest mass of an electron is obtained when an electron is displaced a distance corresponding to the uncertainty of the location of the electron, its (reduced) Compton wavelength, $\lambda_{c}=\hbar / m c$. In the experimentally relevant case of energetic particles in a single crystal, the Lorentz-invariant field parameter $\chi=\gamma \mathcal{E} / \mathcal{E}_{0}$ becomes of order unity, thus the field in the electron rest frame becomes critical. Furthermore, the strong field is of macroscopic extent, as opposed to the case of heavy ion collisions.

Theory shows that the probability per unit length of trident production through a virtual photon (the direct process) in an external field with $\chi \gg 1$ becomes

$$
W_{v}=\frac{13 \alpha^{2} m c \chi(\ln (\chi)-5.46)}{18 \sqrt{3} \pi \hbar \gamma}
$$

i.e. $W_{v} \simeq 18 \chi(\ln (\chi)-5.46) / \gamma\left[\mu \mathrm{m}^{-1}\right]$ [3-6], see also [7]. Likewise, the probability per unit length squared of trident production through a real photon (the sequential process) in an external field for any value of $\chi$ becomes $[5,6]$

$$
\begin{aligned}
W_{r}= & \frac{3 \chi}{16}\left(\frac{\alpha}{\gamma \lambda_{c}}\right) \ln \left(1+\frac{\chi}{12}\right) \times \exp \left(-\frac{16}{3 \chi}\right) \\
& \times\left(1+0.56 \chi+0.013 \chi^{2}\right)^{1 / 6}
\end{aligned}
$$

to an accuracy quoted as better than $4 \%$. Thus, for an electron impinging on a strong crystalline field characterized by $\chi$ of the order one, a significant enhancement of trident production compared to incidence on an amorphous foil should be expected. We show in the following by experiment that this enhancement is indeed present, although it is not as large as the factor of $\simeq 7$, which is predicted by theory, but rather a factor of $\simeq 2$.

The trident process is of high importance e.g. for beamstrahlung (in the general sense, i.e. including emission due to higher-order processes) in the Compact LInear Collider (CLIC) under conceptual planning at CERN, where e.g. the incoherent process $e^{-}+e^{+} \rightarrow e^{-}+e^{+}+e^{-}+e^{+}$has a cross section of several mbarn (the Landau-Lifshitz cross section $28 \alpha^{4} \hbar^{2} \ln ^{3}\left(\gamma^{2}\right) / 27 m^{2} c^{2}$ is tens of mbarn [8]). Also coherence may influence the process in this case, due to the lepton of one bunch interacting with the Lorentzcontracted field of the opposing bunch, resulting in $n_{t} \approx$ $\sigma_{z} W_{v} N_{b}$ (direct) trident events per bunch crossing where 
$\sigma_{z}$ is the bunch length and $N_{b}$ the number of particles in the bunch [9]. Trident events can thus be generated at a rate of several $\mathrm{GHz}$ [10], depending on the chosen bunch length. In fact, beamstrahlung and radiation or particle emission in crystals are closely related processes [11]. Despite the similarities, in the beamstrahlung community trident events typically refer to those mediated by a virtual photon while the sequential events are called "coherent pairs," whereas in the general strong field case and for crystals, tridents-as for the historical three-prong track origininclude both types, see e.g. [5,6]. Based on experience gained from experimental investigations using crystals, extensive simulations are presently being carried out to determine the trident contribution to backgrounds at CLIC [12].

\section{STRONG CRYSTALLINE FIELDS}

Under small angles of incidence to a crystallographic direction in a single crystal — an axis or a plane-the strong electric fields of the nuclear constituents add coherently such that a macroscopic, continuous field with a peak value of the order $\mathcal{E} \simeq 10^{11} \mathrm{~V} / \mathrm{cm}$ is obtained. Fields of order $\mathcal{E}_{0}-5$ orders of magnitude higher — can nevertheless be probed in the rest frame of the electron, exploiting the relativistic invariance of the parameter $\chi=\gamma \mathcal{E} / \mathcal{E}_{0}$ and using electrons with Lorentz factors $\gamma \simeq 10^{5}-10^{6}$ in single crystals.

In the following we show experimentally, that trident processes $e^{-}+\mathcal{E} \rightarrow e^{-}+\mathcal{E}+e^{-}+e^{+}$become significant when the continuous external field $\mathcal{E}$ is "turned on". This happens when the crystal is rotated from a "random" orientation (essentially amorphous configuration) where the fields are screened Coulomb fields, to an axial orientation where the nuclear fields are coherently adding up along the direction of motion of the penetrating particle, such that a field of order $\mathcal{E}_{0}$ is experienced by the electron. See e.g. [6,13] for reviews on strong field effects in crystals.

In order to achieve a significant contribution from the direct process, as compared to the sequential one, the thickness of the crystal must be of the order of the formation length of the photon $[5,6]$. This length is given for real photons by

$$
l_{f}=\frac{2 \gamma^{2} c}{\omega^{*}}
$$

where $\hbar \omega$ is the energy of the photon producing the pair and $\omega^{*}=\omega E /(E-\hbar \omega)$ (see also [13] for a discussion of formation lengths). The frequency under recoil $\omega^{*}$ reduces to $\omega$ for soft photons $\hbar \omega \ll E$ such that $l_{f} \simeq 2 \gamma^{2} c / \omega$. We note that the formation length grows with decreasing photon energy, such that the relative contribution of the direct process can be expected to be most significant at low photon energies, for fixed crystal thickness and energy of the impinging electron.
The formation length for a virtual photon is in a sense an ill-defined concept, since virtual photons are not "formed", but appear only in intermediate stages. It is nevertheless relevant to derive the formation length for a virtual (massive) photon where the square of its energymomentum four-vector $\hbar k^{2}=\hbar k_{\mu} k^{\mu}$ may be different from zero [6]

$$
l_{f v}=\frac{2 \gamma^{2} c}{\omega^{*}} \frac{1}{1+\frac{\hbar^{2} k^{2}}{m^{2} c^{4}} \frac{1+u}{u^{2}}}
$$

where $u=\hbar \omega /(E-\hbar \omega)$ and therefore the emission of a massive, timelike photon (also spacelike virtual photons contribute $[2,14]), k^{2}>0$, may result in a substantial shortening of the formation length.

We emphasize that with our setup, no experimental distinction is possible, between events generated from a virtual photon (direct tridents), and events generated from a real photon (sequential tridents). However, according to theory, both processes are significant under the present experimental conditions.

Calculations showing a drastic increase of trident production in crystals compared to an amorphous material were published more than two decades ago [15] and have recently been refined [16]. As shown in [16], in the amorphous case the ratio of direct to sequential trident production events is expected to be high, ranging from about a factor 7 at $1 \mathrm{GeV}$ positron energy produced from a $180 \mathrm{GeV}$ electron, to 2 at $10 \mathrm{GeV}$ positron energy, both in the case of a $170 \mu \mathrm{m}$ thick Ge target.

\section{KLEIN PARADOX ANALOGUE}

When an electron is impinging on a high electrostatic potential barrier, as is the case in the present experiment, it may penetrate or be reflected. Classically, for electron energies smaller than the barrier height, the electron is always reflected. In nonrelativistic quantum mechanics, an exponentially damped electron tunneling into the barrier is predicted, whereas as shown in 1929 by Oscar Klein [17], in relativistic quantum theory an undamped electroncurrent is always present beyond the barrier, even if it is infinitely high. This is the basis of the "Klein paradox"assured penetration into a classically forbidden region. As later shown by Sauter [18,19]—inspired by a supposition by Bohr-the field required for this to happen is at least the critical field. Today, the Klein paradox is explained by the creation of electron-positron pairs at the boundary.

Previous studies of the Klein paradox have been limited to theory [20-23], e.g. with phenomena analogous to the Klein paradox, possibly observable in graphene $[24,25]$ or with single trapped ions [26]. Such studies have been partly motivated by heuristic arguments linking the Klein paradox, strong field pair production and Hawking radiation from black holes [27-30]. However, a typical general statement on the experimental status has hitherto been 
"... direct tests of the Klein paradox using elementary particles have so far proved impossible" [24], most likely stimulated by the impossibility of producing ultrastrong static electric or magnetic fields. This is also the case here, but our experimental results nevertheless show that pair production does occur for elementary particles in a sufficiently strong electric field, thus putting a Klein paradox analogue into experimental perspective. An important difference between the true Klein paradox and the trident production described here, is that in the former case the invariant "inherent" normalized field strength $\Xi=F_{\mu \nu}^{2} / \mathcal{E}_{0}^{2}=2\left(\vec{B}^{2}-\overrightarrow{\mathcal{E}}^{2}\right) / \mathcal{E}_{0}^{2}$ is comparable to one, while it is close to zero in the latter (discussions addressing similar invariants seem to be missing in most other Klein paradox parallels). In both cases, the second relativistic invariant $\chi$ defined from $\chi^{2}=\left(F_{\mu \nu} p^{\nu}\right)^{2} / m^{2} c^{4} \mathcal{E}_{0}^{2}$ is about one, while the third invariant $\Gamma=e_{\lambda \mu \nu \rho} F^{\lambda \mu} F^{\nu \rho} / \mathcal{E}_{0}^{2}=$ $8 \overrightarrow{\mathcal{E}} \cdot \vec{B} / \mathcal{E}_{0}^{2}$ is zero.

\section{EXPERIMENT}

The experiment was performed in the $\mathrm{H} 4$ beam line of the CERN Super Proton Synchrotron using tertiary beams of electrons with energies of nominally 125, 180 and $210 \mathrm{GeV}$. In Fig. 1 we give a schematical overview of the active elements in the setup of the experiment. The incident electron beam was defined by two scintillator counters, $\mathrm{SC} 1$ and $\mathrm{SC} 2$, in anticoincidence with a $12 \mathrm{~mm}$ hole scintillator, $\overline{\mathrm{ScH}}$, and the beam profile and angles were measured on an event-by-event basis in the first two drift chambers (DC1 and DC3). This was followed by a magnetic dipole of the type MBPL which was only used for measurements with photons impinging on the target, i.e. investigations of background processes as described below. The targets of thicknesses $170 \mu \mathrm{m}$ and $400 \mu \mathrm{m} \mathrm{Ge}\langle 110\rangle$ were mounted on a precision goniometer with $1.7 \mu \mathrm{rad}$ stepsize. Downstream the target, a pair spectrometer (PS) consisting of two drift chambers (DC5 and DC6) and a magnetic dipole of the type MDX, allowed the reconstruction of the momentum of the produced particles.

Furthermore, a solid-state-detector (SSD), allowed the identification of events where a pair was produced by the impinging electron, by measuring the restricted energy loss which is proportional to the number of charged particles in

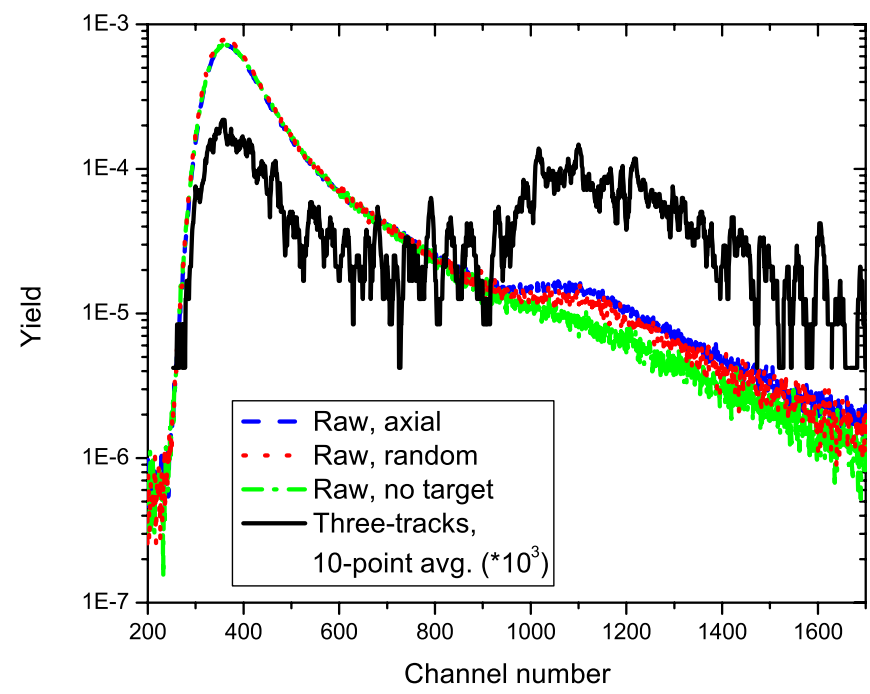

FIG. 2 (color online). SSD spectra for the $170 \mu \mathrm{m} \mathrm{Ge}\langle 110\rangle$ crystal obtained under axially aligned conditions (blue dashed line), under "random" alignment (red dotted) and without target (green dash-dotted). The full line (with an average over 10 channels and multiplied by $10^{3}$ ) shows events in the SSD which have three hits in either DC5 or DC6 and 3 or 2 in the other. The peak around channel 1100 is identified as trident events.

the event, see Fig. 2. The setup was optimized to get maximum detection capability for pairs with lepton energies in the interval $0.5-5 \mathrm{GeV}$, since these pairs are the most abundant and interesting due to the large formation lengths. Under the condition of emission up to a total of $10 \mathrm{GeV}$, the original $E>100 \mathrm{GeV}$ electron impinging on the target loses relatively little energy and proceeds almost undisturbed by the magnetic field in the MDX towards the central lead-glass block. The emitted particles (electrons and positrons) are finally intercepted in the two outermost lead-glass detectors (LG). These LG detectors cover the sensitive region of DC5 and DC6, except the central $22 \mathrm{~mm}$ where the original electron, and a possible emitted photon, can pass towards the central LG. Thus, the two outermost LG intercept the produced electrons and positrons that are simultaneously momentum analyzed by the deflection imposed by the MDX and registered in DC5 and DC6. The setup therefore has redundancy in that the momentum and energy of the produced particles - which at these energies are essentially equal - can be measured.

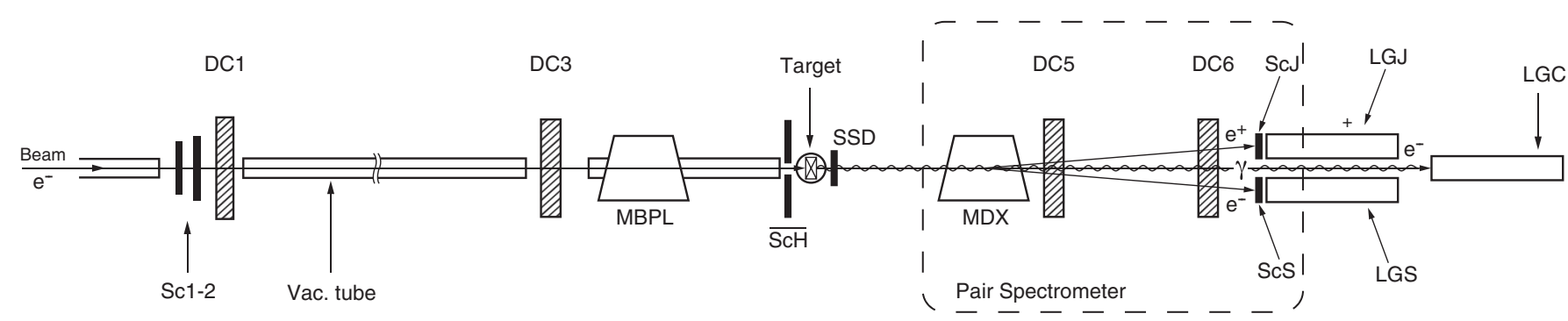

FIG. 1. A schematical drawing — not to scale_ of the setup used in the experiment. The total length is about $65 \mathrm{~m}$. 
The setup was calibrated by use of electrons of nominal energies $10,20,25,50,150,180$ and $210 \mathrm{GeV}$ with the MDX on (as for running conditions- $\int B d l=0.1 \mathrm{Tm}$ - to calibrate DC5 and DC6 for the momentum analysis) and off (to calibrate the downstream LG elements which were mounted on a movable table such that all three elements were exposed one at a time to the beams).

The target was an essentially perfect germanium crystal, previously used for radiation emission studies [31]. The thickness was chosen as a compromise between expected yield and a high ratio of direct (virtual intermediate photons) to sequential (real intermediate photons) trident production. That ratio is, as mentioned, expected to be significant for crystal thicknesses comparable to the formation length, Eq. (3). Thus, for $\hbar \omega=1 \mathrm{GeV}$ and $E=$ $210 \mathrm{GeV}$, we get $l_{f}=66 \mu \mathrm{m}$, but as this is an estimate only, and since crystals with thicknesses below $\simeq 100 \mu \mathrm{m}$ are not sufficiently rigid to be stable we chose a $170 \mu \mathrm{m}$ Ge $\langle 110\rangle$ crystal (and a supplementary $400 \mu \mathrm{m} \mathrm{Ge}\langle 110\rangle$, which was however not as accurately aligned according to the offline analysis, see below). A reasonably accurate average value of $\chi$ for $\mathrm{Ge}\langle 110\rangle$ at room temperature is 1.0 at $210 \mathrm{GeV}$ [6], i.e. the field is of critical magnitude in the rest frame of the impinging electron. This strong field effect is present for all particles within the so-called Baier angle $\Theta_{B}=U_{0} / m c^{2} \simeq 0.5 \mathrm{mrad}$ for $\mathrm{Ge}\langle 110\rangle$, where $U_{0}$ is the amplitude of the crystal transverse potential. The critical angle for channeling - proportional to $1 / \sqrt{p v}$ - in Ge $\langle 110\rangle$ is $0.7 \mathrm{mrad}$ at $1 \mathrm{GeV}$. Channeled electrons and positrons suffer very different energy losses, due to the attraction to or repulsion from the string of nuclei composing the axis.

Since a photon may be generated by the initial electron-in background materials or in the target itself-also a measurement of the pair production under similar conditions as the trident production was investigated.

In the pair production experiment, which preceded the trident experiment, two extra drift chambers were included. One of these, DC2, was placed between DC1 and DC3 and $4 \mathrm{~mm}$ of amorphous $\mathrm{Cu}$ (28\% of a radiation length, $X_{0}$ ) was positioned right downstream this chamber to generate photons. The other extra drift chamber, DC4, was situated downstream the MBPL for tagging of the photons from the electrons deflected in the magnet. In this experiment there was no vacuum in the MBPL magnet and the vacuum in Fig. 1 from the MBPL magnet to $\overline{\mathrm{ScH}}$ was replaced by a $9.6 \mathrm{~m}$ long He tank of diameter $110 \mathrm{~cm}$, allowing for deflection of the electrons.

\section{CRYSTAL ALIGNMENT}

Alignment of the germanium crystals was initially performed via laser reflection off their mirror surfaces, such that the surface normal was directed along the nominal beam. Alignment with respect to the beam was then done by use of $20 \mathrm{GeV}$ electrons and the MDX at maximum field
( $\int B d l \simeq 0.5 \mathrm{Tm}$ ), displacing the array of LG elements to intercept the initial electrons in one of the outermost blocks while letting the photons radiated from the crystal proceed to the central lead-glass block. The enhanced radiation emission when the electron penetrates the crystal along a plane or axis gave a signal-to-noise ratio of about 1 to 1 , such that a stereogram enabled a precise identificationwithin $\simeq 25 \mu \mathrm{rad}$ - of the location in angular space of the $\langle 110\rangle$ axis. This alignment procedure was regularly repeated, in particular, when the beam energy was changed. Using DC1 and DC3, the angular direction $\psi$ of e.g. the $180 \mathrm{GeV}$ beam could be confirmed with respect to the $20 \mathrm{GeV}$ beam to be shifted by $\Delta \psi_{x} \simeq 25 \mu \mathrm{rad}$ and $\Delta \psi_{y} \simeq$ $30 \mu \mathrm{rad}$, compared to the RMS angular widths of the beam $\sigma\left(\psi_{x}\right) \simeq \sigma\left(\psi_{y}\right) \simeq 125 \mu \mathrm{rad}$. Any offset could be corrected for in the offline analysis, down to the angular resolution limit of the DCs of about $3 \mu \mathrm{rad}$.

The $170 \mu \mathrm{m}$ thick crystal was essentially perfectly aligned to the beam, as found in the offline analysis, but unfortunately the $400 \mu \mathrm{m}$ thick crystal was by mistake aligned at an angle of about $0.3 \mathrm{mrad}$ to the axis.

The misalignment of the thick crystal is supported by an examination of the dependence of the enhancement on the selection of entrance angle, where the trident enhancement of the thick crystal is not symmetrical around the center of the angular distribution. Similar investigations of the dependencies on entrance angles in both transversal coordinates have been made for the thin crystal, and these graphs show symmetric dependencies of the entrance angle with respect to the center of the angular distribution. There are thus no indications of inaccurate alignment of the thin crystal.

\section{ANALYSIS}

The drift chambers were calibrated using veto scintillators containing many $1 \mathrm{~mm}$ wide parallel slits spaced $3 \mathrm{~mm}$ apart, placed up- or downstream in the path of the beam and near the relevant chambers. Straight beams were aimed at these scintillators allowing precise calibration of the DC time-to-digital-converter signals, as previously described in [32].

The calibration of the DCs was tested through the correlations between signals from adjacent chambers. This method provided corrections to the calibration of, in particular, the PS drift chambers, 5 and 6 . A series of calibration iterations yielded a final FWHM resolution $\approx$ $100 \mu \mathrm{m}$ in the central part of the beam. This is as expected for the type of drift chamber utilized.

\section{A. Pair spectrometer}

The MDX magnet was calibrated using direct electron beams of known energies of 10,25 and $50 \mathrm{GeV}$ in the PS with the MDX magnet at positive and negative polarity. This provided data for determining the integrated field, as well as the PS resolution as seen in Fig. 3. 


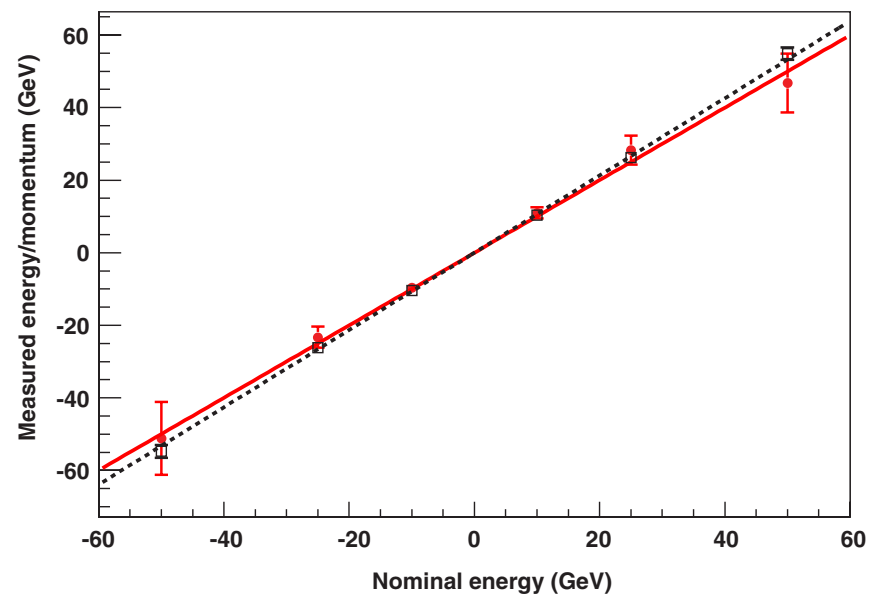

FIG. 3 (color online). Comparison between the nominal momentum of the tertiary electron beam and the momentum/energy measured in the PS (open symbols, dotted line) LGC (full symbols, solid line) including least-squares linear fits.

By extrapolation, assuming a momentum dependence of the uncertainty of

$$
\frac{\delta p}{|p|}=\sqrt{A^{2}+B^{2} p^{2}}
$$

an estimate of the uncertainty at smaller PS momenta was obtained, where $A=\delta \theta_{\text {mul }} / \theta$ depends on multiple scattering and $B$ on the chamber resolutions.

A fit to uncertainties obtained for 10,20 and $50 \mathrm{GeV}$ at both polarities of the MDX, gave a value of $A=0.12 \pm$ 0.02 . Besides multiple scattering this includes the rather large momentum spread of the initial beam, a side effect of the requirement of high intensity and good parallelism. The value of $B=0.025(\mathrm{GeV} / c)^{-1}$ corresponded to a combined chamber resolution for momentum determination of approximately $250 \mu \mathrm{m}$ which is in good agreement with the expected resolution of three chambers in combination. The relative momentum uncertainty is thus approximately $10-15 \%$ for $p$ in the range $0.5-5 \mathrm{GeV}$.

\section{B. Identification of tridents}

In the analysis, a trident event was identified as one in which there were either three hits in both DC5 and DC6 (" $3+3$ events") or two hits in one of these chambers and three hits in the other (" $2+3$ events"). Furthermore, a restriction on the SSD spectrum was imposed, requiring a signal corresponding to three charged particles (events above channel 700 in Fig. 2). The $2+3$ events were about $65 \%$ of the total accepted events and are a result of the DCs not having true multihit capability, i.e. two hits close in drift time on the same side of the anode-wire are identified as one. About half of the $2+3$ events are due to missing detection in DC5 and the other half due to missing detection in DC6. In all cases it was required that the central LG detected a particle with energy at least $5 / 9$ of the initial energy.

Since the sought trident reaction is a higher-order QED process, several other processes which yield a comparable or higher number of events must be filtered from the analysis. A key point in the trident selection was the calculation of the vertex position within the MDX magnet which was required to agree with expectations from the incident particle track.

\section{Vertex calculation}

Tracks between the hits in DC5 and DC6 were reconstructed and the resulting vertex in the MDX was verified to be coincident with the magnetic separation point expected from the incident track generated from DC1 and DC3. Likewise, energy and momentum balance was verified. The sign of the charge of the particles in DC5 and DC6 was determined by comparing the position in these chambers with the projection of the incident electron track. In cases where three hits were recorded in DC5 and DC6, events where at least one positive and one negative particle were identified were accepted. It was assumed that the central hit in these chambers was from the incident particle while the two other hits were from a produced pair. The transversal vertex position was hence determined by

$$
x_{v}=\frac{\left(x_{6+}-x_{5+}\right)\left(x_{5+}-x_{5-}\right)}{x_{6-}-x_{5-}-x_{6+}+x_{5+}}+x_{5+}
$$

which is the $x$ coordinate where the positron and electron tracks cross. The indices refer to the chamber in which the hit was recorded and the charge of the particle. In the $z$ direction, the vertex coordinate was

$$
z_{v}=\frac{x_{5+}-x_{5-}}{x_{6-}-x_{5-}-x_{6+}+x_{5 p}}\left(z_{6}-z_{5}\right)+z_{5}
$$

In order to improve detection efficiency, " $3+2$ " and " $2+3$ " events were included. The most reliable of the possibilities was where the momentum of the produced lepton was calculated from the positron momentum. If calculated from the electron momentum, the possibility that the particle was a $\delta$ electron from the target or from the SSD must be considered.

For the ' $3+2$ ' events, the particles detected in the chamber with two hits were practically always the incident electron and one of the produced leptons. Hence, $x_{v}$ was in this case calculated from the incident electron and the positron [33] tracks. In Fig. 4 it is seen that there is strong correlation between the calculated vertex position from the PS, $x_{v}$, and the expected position in the center of the MDX magnet from the incident electron track, $x_{p}$. A fit to the profile of Fig. 4 confirmed that the slope is indeed very close to unity.

The correlation seen in Fig. 4 provided a very stringent test for the identification of trident events by demanding 


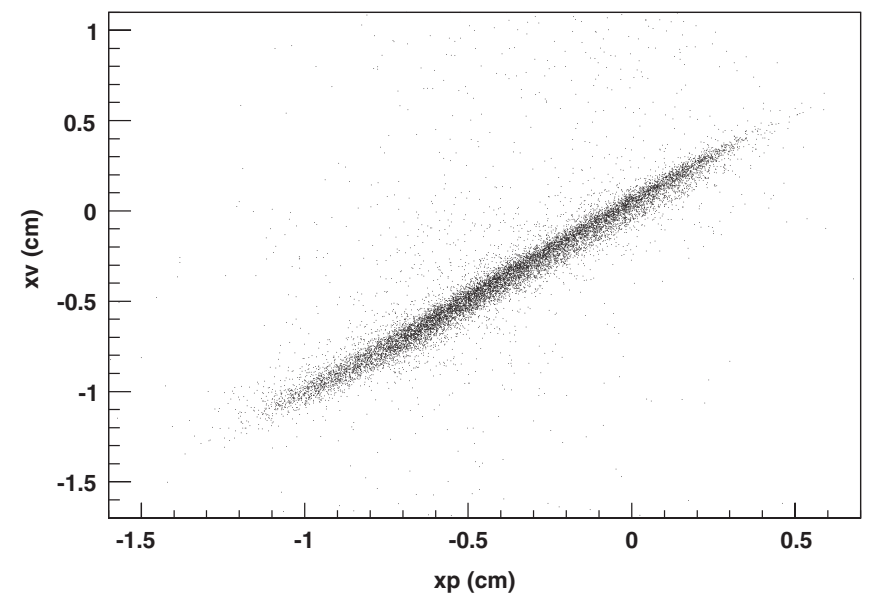

FIG. 4. Correlation between projection of electron track into the center of the MDX magnet, $x_{p}$, and $x_{v}$.

that $x_{v}$ should be no further than $3.0 \mathrm{~mm}$ from the incident electron projection position.

\section{Vertex width}

In order to assess the precision of the determination of the vertex position, a central portion of the $x_{v}, x_{p}$ plot in Fig. 4 was selected. A slice of $x_{p}$ values was selected, and the size of this slice was decreased linearly while the RMS width of the corresponding $x_{v}$ measurement was recorded. A linear extrapolation to zero slice size was done to extract a RMS $x_{v}$ width of $0.64 \mathrm{~mm}$. This width is acceptable considering multiple scattering and the fact that $x_{v}$ was determined by measuring 4 transversal coordinates with a resolution of each $\sim 0.15 \mathrm{~mm}$. In the $z$ direction the vertex position found was broader due to the small deflection angle. The large majority of the trident events were however located inside the gap of the MDX magnet in the analysis, as seen in Fig. 5. In the analysis, a quite nonrestrictive cut in $z_{v}$ of $5800 \mathrm{~cm}<z_{v}<5900 \mathrm{~cm}$ was employed.

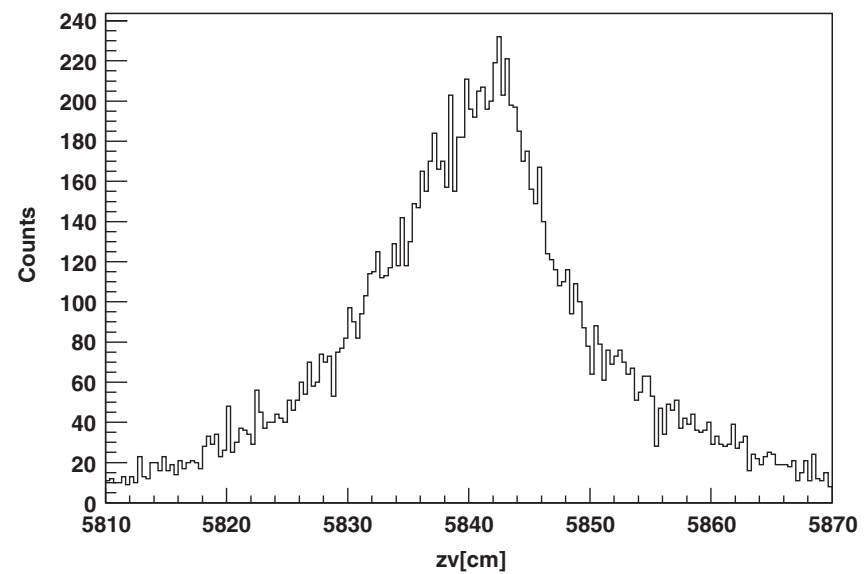

FIG. 5. Distribution of $z$-vertex coordinates. The MDX was located with its center at $z=5840 \mathrm{~cm}$, with a length of $40 \mathrm{~cm}$.

\section{Calculation of lepton momentum}

The identification of leptons in the PS provided the possibility to determine their individual momenta from

$$
p_{e^{+}}=\frac{B l}{\tan ^{-1}\left(\frac{x_{6+}-x_{5+}}{z_{6}-z_{5}}\right)-\theta_{\text {in }}}
$$

As seen in Fig. 6 there is good agreement between the measurement of lepton energy in the relevant calorimeter (in this case the calorimeter block LGJ) and the calculated momentum. The discrepancy at low PS momentum is mostly due to the fact that the geometry does not allow the calorimeter to measure energies lower than $1 \mathrm{GeV}$. Furthermore, a small discrepancy arises due to the fact that the calorimeters were calibrated with minimum direct beam energies of $10 \mathrm{GeV}$. Thus, the lead-glass energy serves only as a qualitative confirmation of the PS working correctly.

\section{Pair production analysis}

As mentioned above, the pair production data served as support for the trident analysis but also as a confirmation of usability of the setup. In the analysis of the data, two particles on either side of the projected hit of the photon in the PS drift chambers were required for an event to be counted.

In analogy to the trident experiment, the common lepton vertex was found, and cuts were applied to ensure as few false events as possible. The SSD detector was not part of the pair production setup, so the event selection was done on a purely kinematic and calorimetric basis.

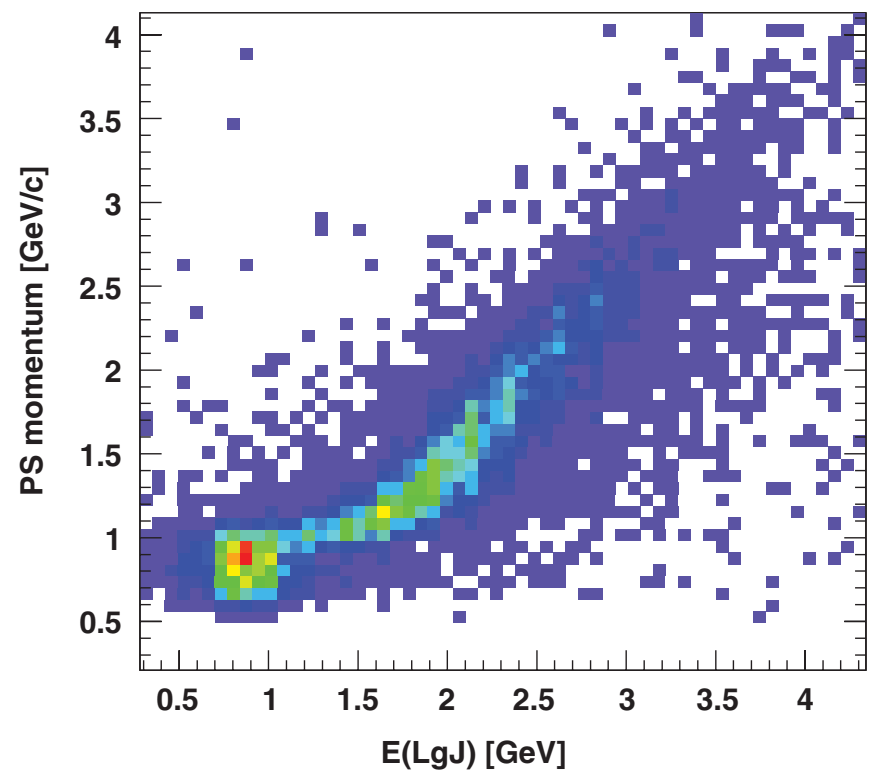

FIG. 6 (color online). Comparison between the LGJ calorimeter and the trident positron momentum found with the PS. 


\section{MONTE-CARLO SIMULATIONS}

In order to evaluate the detection efficiencies for the trident and pair production experiments, a Monte-Carlo simulation was performed for the amorphous case (random alignment). The Monte-Carlo code included bremsstrahlung emission and pair production according to the BetheHeitler mechanisms, multiple Coulomb scattering, drift chamber inefficiencies due to multiple hits and the measured geometry of the setup. Apart from the limitations in multihit capability, the simulation did not include the cell structure of the drift chambers. Therefore, small variations due to an inhomogeneous detection efficiency as a function of momentum was not accounted for.

\section{A. Simulation of the pair production experiment}

In the simulation of the pair production experiment, the $4 \mathrm{~mm}$ thick $\mathrm{Cu}$ conversion target produced photons which converted in a $170 \mu \mathrm{m}$ thick Ge target with the BetheHeitler bremsstrahlung- and pair production probability, respectively. As in the treatment of experimental data, only events with both particles seen in both PS chambers were accepted. A comparison between the simulated and real data is seen in Fig. 7. There is excellent agreement between the experimental data and simulation, over almost 3 orders of magnitude in counted events, showing that the setup is well understood.

\section{B. Trident simulation}

The detection efficiency in the trident experiment was estimated in an enhanced version of the pair production Monte Carlo simulation. Also for the trident experiment a method of detection closely similar to the one employed in the experiment was used. Trident events were generated as in the pair production simulation with a photon conversion

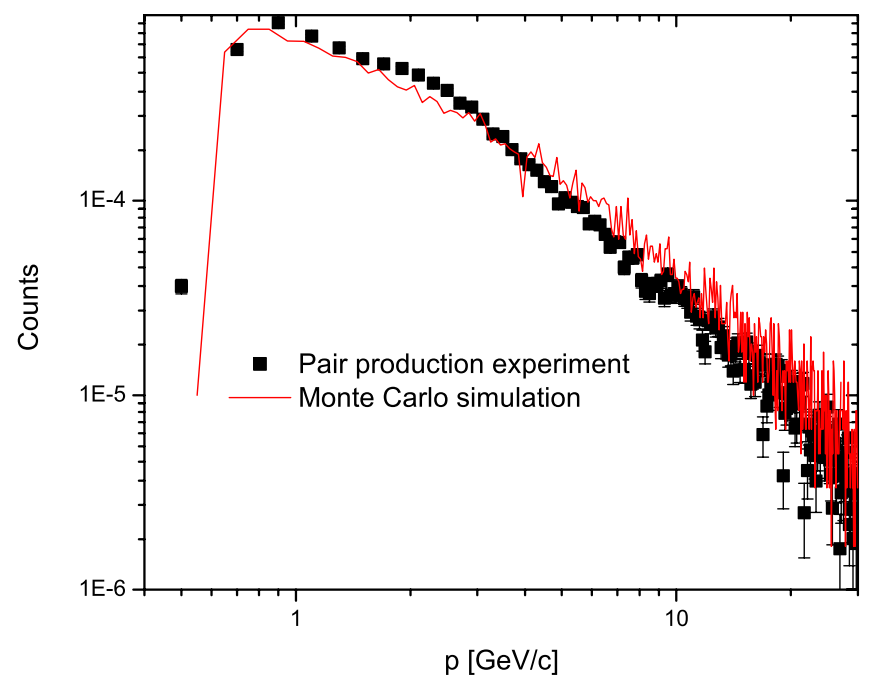

FIG. 7 (color online). Comparison between pair production data and simulation (with a normalized vertical scale).

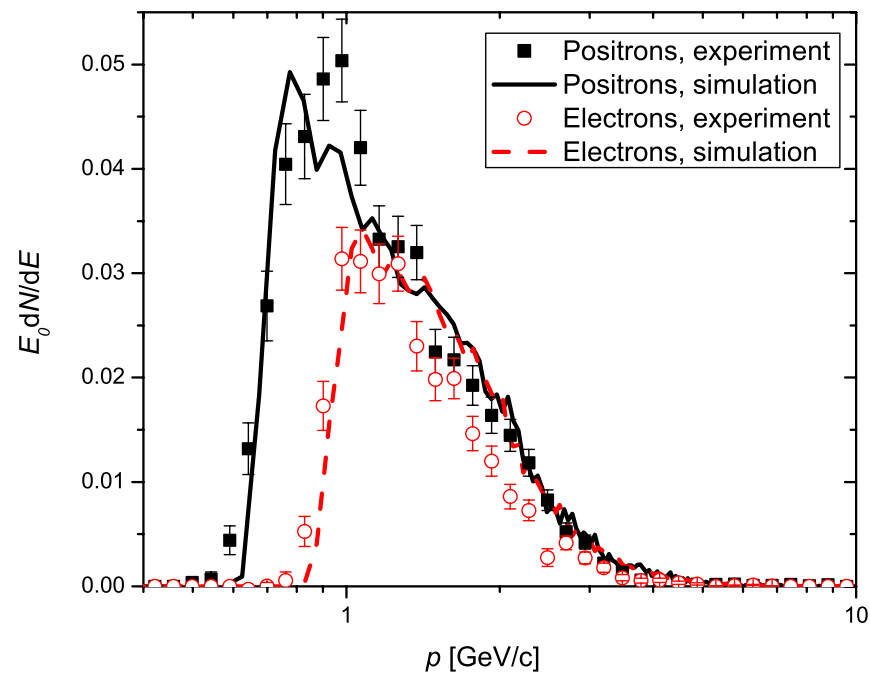

FIG. 8 (color online). Experimental (filled squares) and simulated (full line) trident production in the "random" configuration of the $170 \mu \mathrm{m}$ thick $\mathrm{Ge}\langle 110\rangle$ crystal.

target and an amorphous Ge pair production target, but the incident electron was sent all the way through the setup into the PS, with inclusion of multiple scattering. A minimum time of separation of $180 \pm 60 \mathrm{~ns}$ in the DC readout, corresponding to a minimum transversal distance of $9 \pm 3 \mathrm{~mm}$ between the primary and the produced particles, was implemented in the simulation to obtain good agreement at higher pair energies. This is a realistic number due to dead time in the readout of the anode and does not apply in the pair production experiment where the particles are detected at different anodes. The result of the simulation is seen in Fig. 8, compared to data. The simulation is in good agreement with data, in particular, at low PS momenta where the efficiency is determined mainly by the geometric

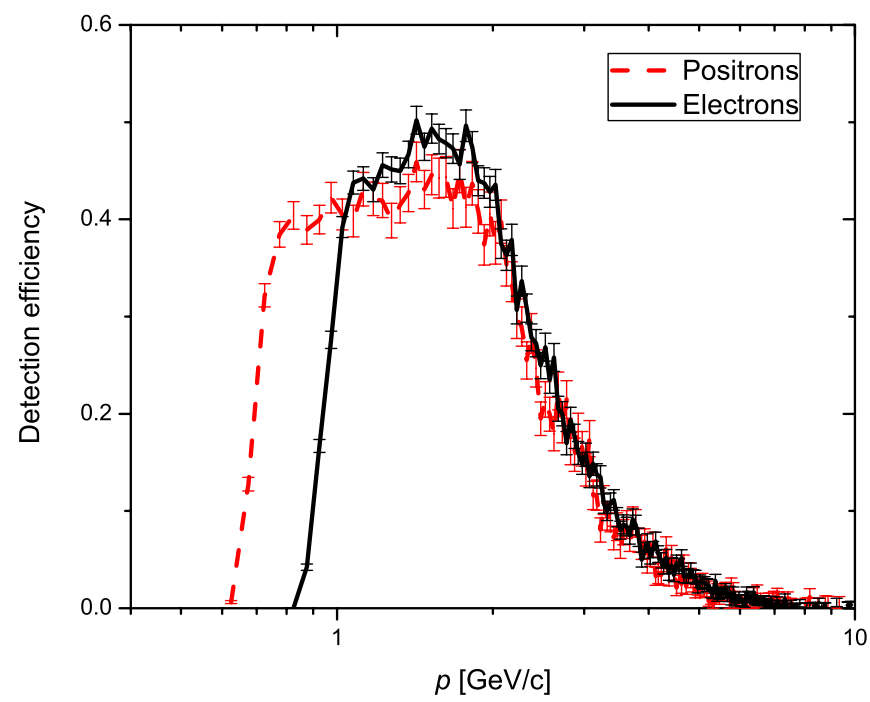

FIG. 9 (color online). Detection efficiency found from simulation. 
parameters of the PS. The simulated detection efficiency, leading to the curves in Fig. 8 is seen in Fig. 9.

The trident detection efficiency - thus shown to be understood by comparison with simulations-was applied to the experimental data for both the "random" and aligned configurations shown below.

\section{SSD cuts}

The SSD was furthermore used to identify events where photons converted to pairs in the space between the SSD and magnet. Such events would otherwise be detected as tridents in the PS. Thus, a cut in the number of minimum ionizing particles seen in the SSD was applied. As shown in Fig. 2 the kinematic selection did isolate the trident events rendering a cut in the SSD spectrum possible. This cut was made at channel 900 based upon an analysis as in Fig. 10 where the enhancement as a function of cut position in the SSD spectrum is seen.

The lack of true multihit capability in the drift chambers made an analysis of the $y$ coordinates of the particle tracks impossible, since it was not known to what $x$ coordinate a given $y$ coordinate belonged. It was however verified that the distribution of $y$ coordinates was in agreement with expectations, and the number of detected $y$ coordinates for a given event was in the analysis demanded lower than 4 in both PS chambers.

\section{Accounting for additional photons}

The SSD cut was an important one to account for converting photons. However, the introduction of this detector to the setup provided extra material for multiple scattering and further reactions. As an estimate of this contribution, a calculation was done based on the Bethe-Heitler pair

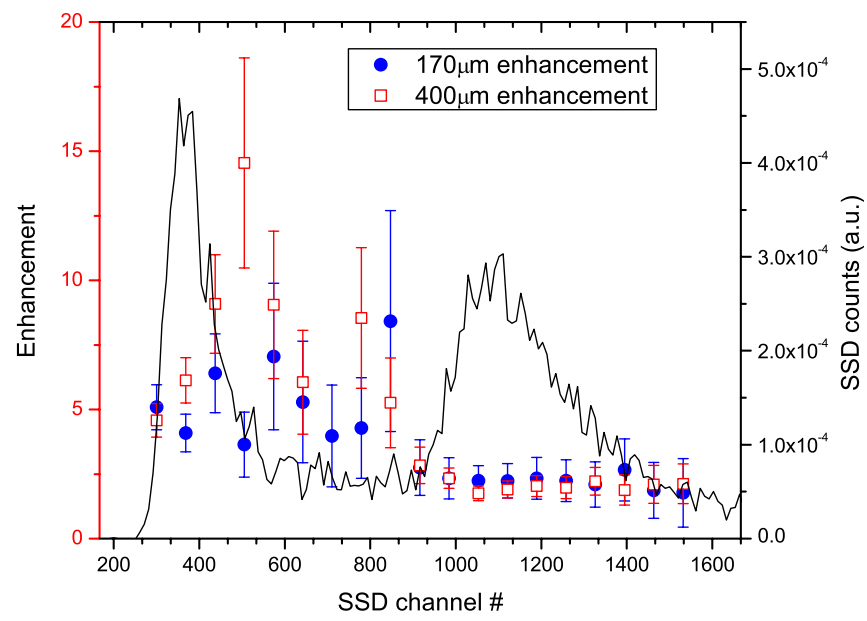

FIG. 10 (color online). Enhancements within narrow windows of SSD channels for the $170 \mu \mathrm{m}$ (filled dots) and $400 \mu \mathrm{m}$ (open squares) thick crystals. For comparison, the SSD spectrum for the $400 \mu \mathrm{m}$ thick crystal at axial alignment and with all trident selection cuts is shown as a line. production, assuming that all bremsstrahlung photons converting in the SSD detector would be seen as three charged particles. Since most of this contribution was subtracted with the background, the only remaining photons were the ones formed in the target. When the Ge crystal was in "random" (amorphous configuration), the contribution of these photons to false trident events was

$$
\frac{d N_{\text {false }}}{d E_{+}}=\int_{E_{+}}^{E_{0}} \frac{d N_{\text {pair }}}{d E_{+}} \frac{d N_{\text {phot }}}{d \hbar \omega} d \hbar \omega,
$$

where $E_{+}$is the energy of the positron in the pair and $E_{0}$ is the incident electron energy. $d N_{\text {pair }} / d E_{+}$is the number of Bethe-Heitler pairs created in the SSD and air between target and SSD, $d N_{\text {phot }} / d \hbar \omega$ is the number of BetheHeitler photons created in the amorphous Ge target. This contribution is seen along with recorded data in Fig. 11 and is clearly not negligible.

A similar estimate of the photon contribution in the case where the crystal was aligned was needed. As in [34]
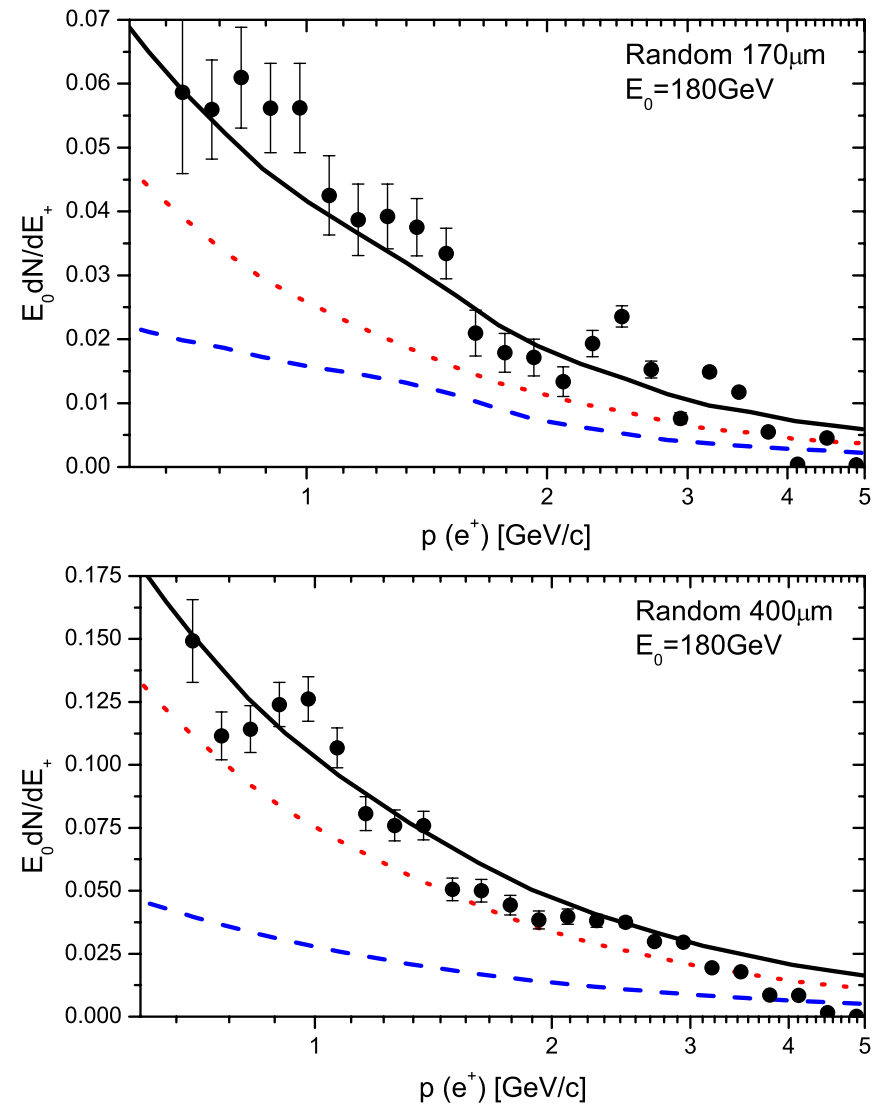

FIG. 11 (color online). Trident positron spectra for $170 \mu \mathrm{m}$ and $400 \mu \mathrm{m}$ thick Ge crystals in the "random" orientation. The energy of the primary particle is $E_{0}=180 \mathrm{GeV}$. The dashed line shows the calculated photon contribution, the dotted line the theoretical expectation according to Baier and Katkov [16] and the solid line shows the sum of these contributions. The filled dots are experimental data corrected for the detection efficiency shown in Fig. 9. 
which is an experimental determination of the bremsstrahlung enhancement in $185 \mu \mathrm{m} \mathrm{Ge}\langle 110\rangle$ at $150 \mathrm{GeV}$, for this calculation we assumed an enhancement of bremsstrahlung photons rising linearly from $\eta=2.2$ at $\hbar \omega=1 \mathrm{GeV}$ to $\eta=4.1$ at $\hbar \omega=20 \mathrm{GeV}$. This means that Eq. (6) was modified to

$$
\frac{d N_{\text {false }}}{d E_{+}}=\int_{E_{+}}^{E_{0}}\left(\frac{\hbar \omega-1 \mathrm{GeV}}{10 \mathrm{GeV}}+2.2\right) \frac{d N_{\text {pair }}}{d E_{+}} \frac{d N_{\text {phot }}}{d \hbar \omega} d \hbar \omega .
$$

Again, this contribution is non-negligible in the absolute rates, but is of marginal importance to the enhancement, as discussed below.

The opposite case, where an increase is due to enhancement of the pair production process, following the emission of a photon along the crystal axis, is included in the theory as "sequential" events.

\section{RESULTS AND DISCUSSION}

\section{A. Spectra at random alignment}

The spectra at "random" crystal alignment are seen in Fig. 11. They are in very good agreement with the calculations by Baier and Katkov [16], taking into account the extra contribution from photons produced. The convincing agreement shown in Fig. 11 gives credibility to the experimental data obtained for an aligned crystal, since essentially the only difference consists in a rotation of the target (to an orientation that is coincident with the beam direction for the $170 \mu \mathrm{m}$ thick Ge crystal, within an angle shown to be a factor $\sim 20$ smaller than the relevant strong field angle $\Theta_{B}$ ).

\section{B. Spectra at axial alignment}

As seen in Fig. 12 the calculated photon contribution is a relatively small correction for both the aligned $170 \mu \mathrm{m}$ thick Ge crystal and for the $400 \mu \mathrm{m}$ thick Ge crystal at an angle of $\simeq 0.3 \mathrm{mrad}$ to the axis. The experimental data points tend to follow the shape of the theoretical curve according to Baier and Katkov [35], but the absolute scales are not identical. The experiment gives less than theory predicts, in particular, for the high positron energies. For the poor agreement between theory and experiment for the $400 \mu \mathrm{m}$ thick crystal, the discrepancy can only be partly explained by the misalignment of $\simeq 0.3 \mathrm{mrad}$, since the Baier angle is almost a factor 2 larger, $\Theta_{B} \simeq 0.5 \mathrm{mrad}$ for $\mathrm{Ge}\langle 110\rangle$.

In contrast to the random spectra shown in Fig. 11, where the factor 2.35 increase in thickness gives an increase of a factor between 2.8 and 3.1 in theory, the aligned spectra show a theoretical increase of a factor between 5.0 and 5.3. In both cases the calculated photon contribution has been neglected, while the inclusion of it would reduce the random factors to 2.4-2.8 but only marginally in the aligned case. The differing factors is due to differences in the relative contributions of the direct and sequential pro-
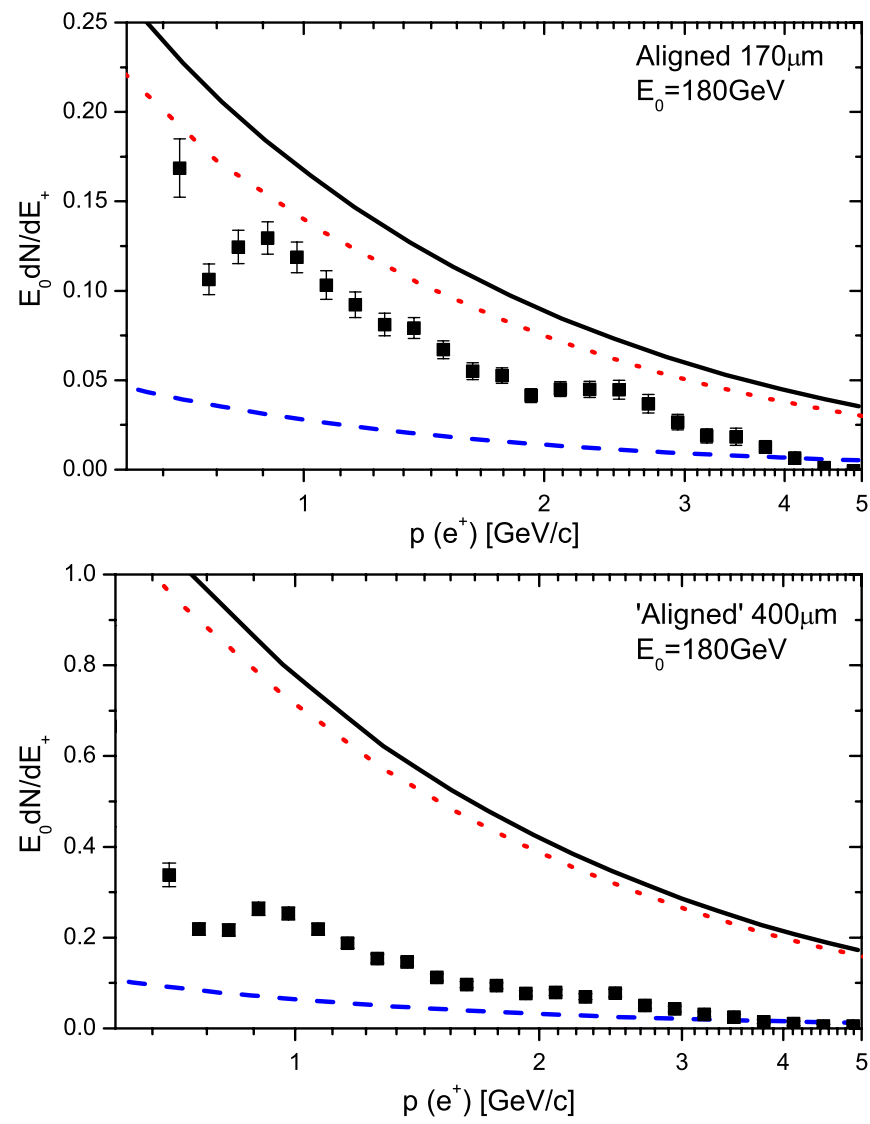

FIG. 12 (color online). Trident positron spectra for the $170 \mu \mathrm{m}$ thick Ge crystal in the $\langle 110\rangle$ axial orientation (upper graph) and for the $400 \mu \mathrm{m}$ thick Ge crystal at an angle of $\simeq 0.3$ to the axis (lower graph). The energy of the primary particle is $E_{0}=180 \mathrm{GeV}$. The dashed line shows the calculated photon contribution, the dotted line the theoretical expectation for precise alignment according to Baier and Katkov [35] and the solid line shows the sum of these contributions. The filled squares are experimental data corrected for the detection efficiency shown in Fig. 9.

cesses, the former of which are dominant in the random case and most significant for low photon energies (large formation lengths), and the latter are dominant in the aligned case. The experimental values in the random case are in fair agreement with this (given that the calculated photon contribution is a larger fraction of the total at small thickness), with an increase of a factor $2.1 \pm 0.3$, depending on the positron momentum. But in the aligned case the data do not show the same trend with an increase of only about a factor 2, partly due to the poor alignment of the thick crystal.

\section{Enhancements}

The enhancements for $180 \mathrm{GeV}$ incident electrons

$$
\eta=\frac{\left(d N / d E_{+}\right)_{\mathrm{ax}}-\left(d N / d E_{+}\right)_{b g}}{\left(d N / d E_{+}\right)_{\mathrm{ran}}-\left(d N / d E_{+}\right)_{b g}}
$$


are seen in Fig. 13, compared to the calculated values of Baier and Katkov [35]. We emphasize that inefficiencies can be neglected to first order in a comparison of spectra obtained under axially aligned conditions to "random" alignment, and that the good agreement with expectations obtained for the amorphous case provides a strong basis for the interpretation of the experiment under aligned conditions. Subtraction of the additional photon contributions calculated by Eq. (7) in the numerator and Eq. (6) in the denominator, does not significantly change the enhancement, given the statistical uncertainty. This is reassuring since the calculation of the photon contribution only serves as an estimate of the maximum number of false trident events. The data sets presented below do therefore not include this subtraction. The enhancement is approximately a factor of 2-3 smaller than theoretically expected for the $170 \mu \mathrm{m}$ thick crystal and the enhancement of the improperly aligned $400 \mu \mathrm{m}$ crystal is approximately the same as for the thin one.
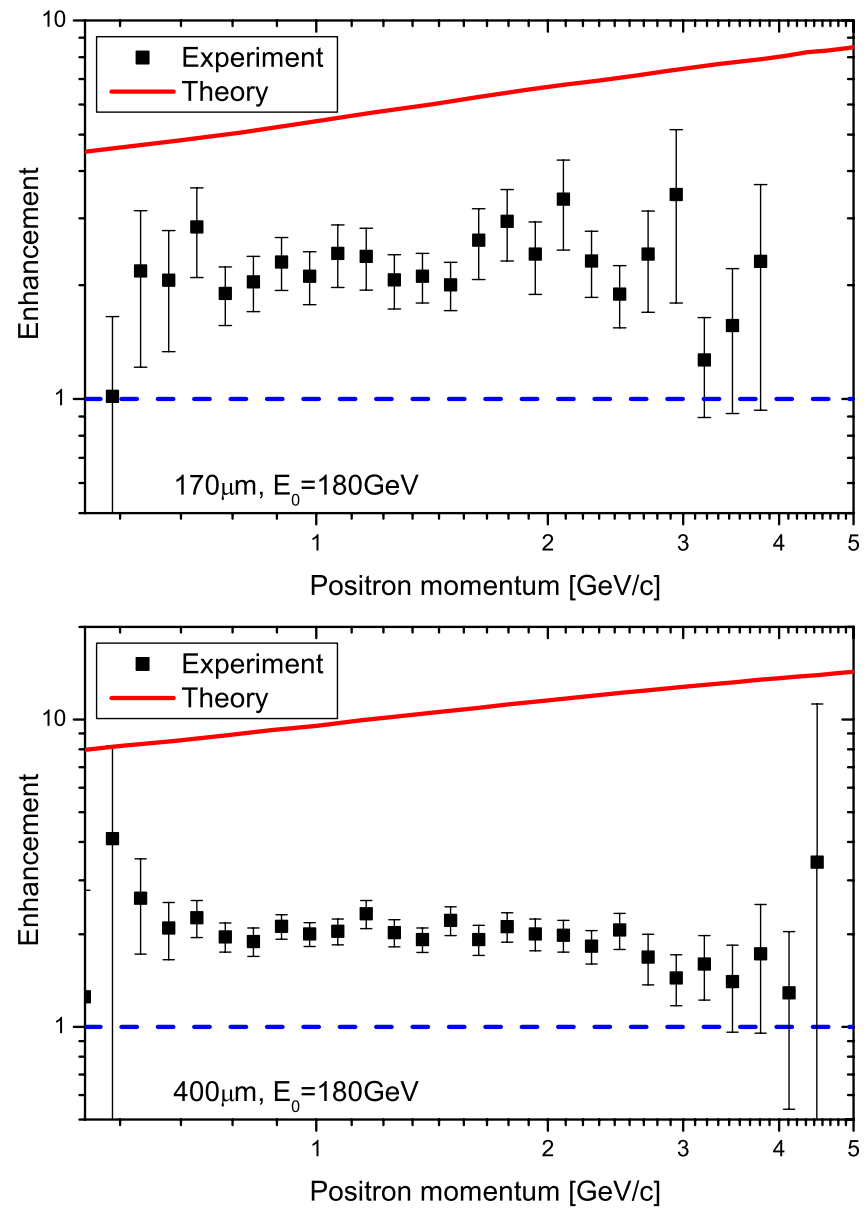

FIG. 13 (color online). Enhancements for the $170 \mu \mathrm{m}$ thick crystal and the $400 \mu \mathrm{m}$ thick crystal, compared to theory according to [35].

\section{Electrons vs positrons and other energies}

Also shown in Fig. 14 with open circles is the differential enhancement as a function of the momentum of the produced electrons for $180 \mathrm{GeV}$ electrons penetrating the $170 \mu \mathrm{m}$ and $400 \mu \mathrm{m}$ Ge $\langle 110\rangle$ crystals. The nice, almost exact agreement between these two modes of detection and analysis, focusing on the produced electron or positron, respectively, shows that systematic uncertainties affecting the results are likely to be small. Furthermore, it shows that effects such as energy loss of the produced particles in the remaining part of the crystal downstream the production point, is likely to be small as electrons tend to suffer much more energy loss close to the axis than positrons. A similar conclusion is reached for the $400 \mu \mathrm{m}$ thick crystal.

Though the detection efficiency for the two methods had low-momentum cutoffs at different momenta, the magnitude of the enhancements thus prove to be the same for both detection methods. It can therefore be inferred that,
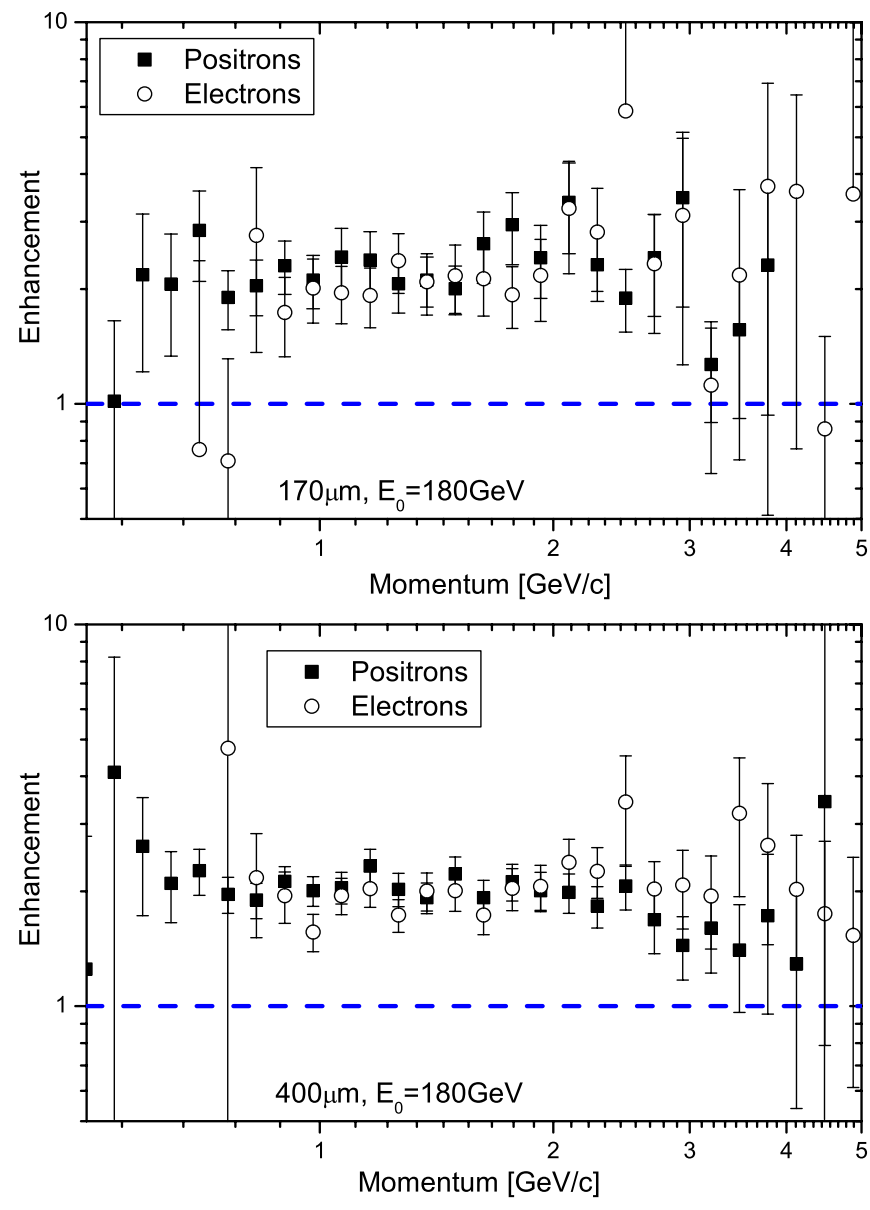

FIG. 14 (color online). Differential enhancement of trident production as a function of the momentum of the produced positron (filled squares) and as a function of the momentum of the produced electron (open circles). The points show results obtained for $180 \mathrm{GeV}$ electrons penetrating a) a $170 \mu \mathrm{m} \mathrm{Ge}$ $\langle 110\rangle$ crystal aligned axially and b) a $400 \mu \mathrm{m} \mathrm{Ge}\langle 110\rangle$ crystal at an angle of $\simeq 0.3 \mathrm{mrad}$ to the axis. 


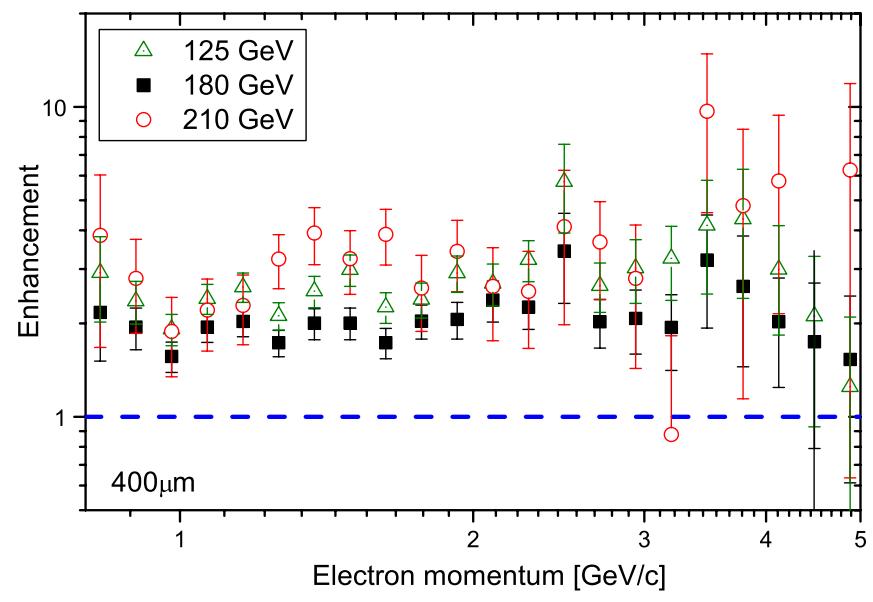

FIG. 15 (color online). Differential enhancement of trident production as a function of the momentum of the produced electron. The points show results obtained for 125 (open triangles), 180 (filled squares) and $210 \mathrm{GeV}$ (open circles) electrons penetrating a $400 \mu \mathrm{m} \mathrm{Ge}\langle 110\rangle$ crystal at an angle of $\simeq 0.3 \mathrm{mrad}$ to the axis.

e.g. $\delta$ electron events were effectively cut away in the analysis by the SSD and the kinematic selection.

Finally, measurements were also performed on the $400 \mu \mathrm{m} \mathrm{Ge}\langle 110\rangle$ crystal for two other initial energies, 125 and $210 \mathrm{GeV}$. However, due to lack of beam time neither "random" spectra nor backgrounds were properly measured at these energies. Assuming the scaling with energy of radiation from an amorphous substance (random or background) is well known, enhancement spectra can nevertheless be constructed for these energies, showing a small trend of increase with rising energy, as seen in Fig. 15 but with statistical uncertainties much too large to reach a firm conclusion.

\section{E. Discrepancy between experiment and theory}

There can be several reasons for the discrepancy between experiment and theory of about a factor 3 in enhancement. One possibility could be that the $170 \mu \mathrm{m}$ thick crystal was not perfectly aligned and therefore gave a smaller enhancement than when properly aligned. We estimate the accuracy of the alignment procedure to be about $25 \mu \mathrm{rad}$, significantly smaller than the relevant angle for strong field effects, $\Theta_{B} \simeq 0.5 \mathrm{mrad}$. This possibility is therefore unlikely for the $170 \mu \mathrm{m}$ thick crystal, but the misalignment for the $400 \mu \mathrm{m}$ thick crystal may be partly responsible for the discrepancy. Also, the quality of the thin crystal, having been used successfully in the past for radiation emission studies, is believed to be very high. Concerning the detector efficiencies and reconstruction algorithms used in the analysis, we find it very reassuring that our data in the "random" (essentially amorphous) configuration is in good agreement with theory. The aligned case uses exactly the same setup and analysis, only the crystal is rotated slightly. We therefore find it hard to see how the experiment could be wrong by as much as a factor 3 .

On the theory side, in [35] it is stated that "The scale of the enhancement in the soft part of created particles spectrum $(z \ll 1)$ is similar to the photon emission enhancement." The reason for this is that the enhancement of photon conversion is close to 1 , being dominated by the incoherent process. Thus, a photon emission enhancement similar to the measured enhancement for the trident process $(\simeq 2-3)$, would support the experiment. Previous measurements of the photon emission enhancement for $150 \mathrm{GeV}$ electrons in a $185 \mu \mathrm{m}$ thick Ge $\langle 110\rangle$ crystal [34] show an enhancement in the soft part that is $\simeq 4$, i.e. similar to our measurements for the trident process (other measurements with $150 \mathrm{GeV}$ electrons in $0.2 \mathrm{~mm}$ thick Ge $\langle 110\rangle$ crystals $[31,36]$ are consistent with this, although the exact numbers are difficult to extract due to the coarse binning in their figures). Moreover, the alignment, based on measurements of enhanced radiation emission, is also consistent with an enhancement of a factor 4 . We therefore tend to believe that the discrepancy, at least for the $170 \mu \mathrm{m}$ thick crystal, is due to oversimplifications in the theoryperhaps not taking the initially channeled, produced electrons and positrons correctly into account-rather than the experiment.

\section{CONCLUSION}

We have observed a significant increase in the number of trident events $e^{-} \rightarrow e^{-} e^{+} e^{-}$for a Ge single crystal under axial alignment, compared to a "randomly" oriented (effectively amorphous) material of the same thickness, using beams of 125,180 and $210 \mathrm{GeV}$ electrons. By this method, we have shown that electrons incident on a strong electromagnetic field produce pairs through the trident process. This observation is in accordance with expectations based on the presence of a critical field in the rest frame of the emitting particle, a phenomenon analogous to that addressed in the Klein paradox. The method takes advantage of the unique combination of strong crystalline fields and high Lorentz factors that produce an ultrastrong electromagnetic field of the order of the critical field, in the rest frame of the emitting particle.

The agreement with theory in the "random" configuration is good over the entire detected range of momentum of the produced particle, whereas in the aligned case a substantial disagreement with theory is observed. We believe this discrepancy to be at least partly due to insufficient account for all relevant crystalline effects in the theory, but our measurements do not necessarily indicate that the theory of strong field trident production-disregarding crystalline effects-is doubtful. Thus, conclusions related to strong field effects in future linear colliders as e.g. CLIC require further experimental studies. 


\section{ACKNOWLEDGMENTS}

We are very grateful for the strong support from P. B. Christensen, P. Aggerholm, and F. Mikkelsen (DPA, Aarhus) as well as the Super Proton Synchrotron operations crew, in particular, I. Efthymiopoulos and B. Chauchaix (CERN) who made an effort in providing the beam. We thank CERN for giving beam time to "alternative programs," i.e. non-LHC related activities.
U.I.U. would like to thank P. Schleper and G. Ridolfi for useful discussions and their professional support in the establishment of NA63 at CERN. Financial support from the Danish Natural Science Research Council (FNU/NICE), the South African National Research Foundation, and Istanbul University Scientific Research Projects (IU BAP 6188) is acknowledged.
[1] M. M. Block, D. T. King, and W. W. Wada, Phys. Rev. 96, 1627 (1954).

[2] B. Grossetete, R. Tchapoutian, D. J. Drickey, and D. Yount, Phys. Rev. 168, 1475 (1968).

[3] V. N. Baier, V. M. Katkov, and V. M. Strakhovenko, Sov. J. Nucl. Phys. 14, 572 (1972).

[4] V. I. Ritus, Ann. Phys. (N.Y.) 69, 555 (1972).

[5] V. N. Baier, V. M. Katkov, and V. M. Strakhovenko, Sov. J. Nucl. Phys. 53, 634 (1991).

[6] V. N. Baier, V.M. Katkov, and V.M. Strakhovenko, Electromagnetic Processes at High Energies in Oriented Single Crystals (World Scientific, Singapore, 1998).

[7] T. Erber, Rev. Mod. Phys. 38, 626 (1966).

[8] V. N. Baier and V. M. Katkov, Phys. Rep. 409, 261 (2005).

[9] K. A. Thompson and P. Chen, in Proceedings of Quantum Aspects of Beam Physics, Monterey, edited by P. Chen (SLAC Report No. SLAC-PUB-7776, 1998).

[10] A. Ferrari, V. Ziemann, R. B. Appleby, and M. D. Salt, Phys. Rev. ST Accel. Beams 12, 021001 (2009).

[11] J. Esberg and U.I. Uggerhøj, J. Phys. Conf. Ser. 198, 012007 (2009).

[12] J. Esberg, D. Schulte, and U. I. Uggerhøj (unpublished).

[13] U. I. Uggerhøj, Rev. Mod. Phys. 77, 1131 (2005).

[14] S. J. Brodsky and S. D. Drell, Annu. Rev. Nucl. Sci. 20, 147 (1970).

[15] J. C. Kimball and N. Cue, Phys. Rep. 125, 69 (1985).

[16] V. N. Baier and V. M. Katkov, JETP Lett. 88, 80 (2008).

[17] O. Klein, Z. Phys. 53, 157 (1929).

[18] F. Sauter, Z. Phys. 69, 742 (1931).

[19] F. Sauter, Z. Phys. 73, 547 (1932).
[20] W. Greiner, B. Müller, and J. Rafelski, Quantum Electrodynamics of Strong Fields (Springer, Berlin, 1985).

[21] H. Nitta, T. Kudo, and H. Minowa, Am. J. Phys. 67, 966 (1999).

[22] P. Krekora, Q. Su, and R. Grobe, Phys. Rev. Lett. 92, 040406 (2004).

[23] R. Giachetti and E. Sorace, Phys. Rev. Lett. 101, 190401 (2008).

[24] M. I. Katsnelson, K. S. Novoselov, and A. K. Geim, Nature Phys. 2, 620 (2006).

[25] A. Calogeracos, Nature Phys. 2, 579 (2006); M. Buchanan, ibid. 2, 721 (2006).

[26] L. Lamata, J. Leon, T. Schätz, and E. Solano, Phys. Rev. Lett. 98, 253005 (2007).

[27] B. Müller, W. Greiner, and J. Rafelski, Phys. Lett. 63A, 181 (1977).

[28] B. R. Holstein, Am. J. Phys. 66, 507 (1998).

[29] B. R. Holstein, Am. J. Phys. 67, 499 (1999).

[30] D. N. Page, New J. Phys. 7, 203 (2005).

[31] R. Medenwaldt et al., Phys. Lett. B 242, 517 (1990).

[32] H. D. Hansen et al., Phys. Rev. D 69, 032001 (2004).

[33] In the case where the momentum of the produced lepton was measured as the electron momentum, the vertex positions were calculated as the crossing between incident and produced electron tracks.

[34] A. Belkacem et al., Phys. Lett. B 177, 211 (1986).

[35] V.N. Baier and V.M. Katkov, Phys. Lett. A 373, 1874 (2009).

[36] R. Medenwaldt et al., Phys. Rev. Lett. 63, 2827 (1989). 